\title{
The Volume of a Local Nodal Domain
}

\author{
Dan Mangoubi
}

\begin{abstract}
Let $M$ either be a closed real analytic Riemannian manifold or a closed $C^{\infty}$-Riemannian surface. We estimate from below the volume of a nodal domain component in an arbitrary ball, provided that this component enters the ball deeply enough.
\end{abstract}

\section{Introduction}

\section{$1.1 \quad$ Main Results}

Let $(M, g)$ be a closed $C^{\infty}$-Riemannian manifold of dimension $n$. Let $\Delta=$ - div o grad be the Laplace-Beltrami operator on $M$. We consider the eigenvalue equation

$$
\Delta \varphi_{\lambda}=\lambda \varphi_{\lambda}
$$

For any $\lambda$-eigenfunction $\varphi_{\lambda}$ the null set $\left\{\varphi_{\lambda}=0\right\}$ is called the $\varphi_{\lambda}$-nodal set and any connected component of the set $\left\{\varphi_{\lambda} \neq 0\right\}$ is called a $\lambda$-nodal domain.

The Faber-Krahn Inequality (EK96]) shows that the volume of any $\lambda$ nodal domain $\mathcal{A}_{\lambda}$ is $\geq C /(\sqrt{\lambda})^{n}$. Donnelly and Fefferman initiated in [DF90] the study of a local version of the Faber-Krahn inequality. Namely, they gave a lower bound on the volume of local nodal domains:

Theorem 1.2 ([DF90, CM91, Lu93]). Let $\varphi_{\lambda}$ be a $\lambda$-eigenfunction. Let $B$ be an arbitrary metric ball in $M$, and let $\Omega_{\lambda}$ be a connected component of $\left\{\varphi_{\lambda} \neq 0\right\} \cap B$. If $\Omega_{\lambda} \cap \frac{1}{2} B \neq \emptyset$ then

$$
\frac{\left|\Omega_{\lambda}\right|}{|B|} \geq \frac{C_{1}}{(\sqrt{\lambda})^{\alpha(n)}(\log \lambda)^{4 n}},
$$

where $\alpha(n)=4 n^{2}+n / 2$. 
Notations. In the above theorem and throughout the paper $r B$ denotes a concentric ball whose radius is $r$ times that of $B . C_{1}, C_{2}, \ldots$ denote constants which depend only on the metric $g$. The enumeration of constants is different in each section.

Donnelly and Fefferman note that the estimates above are clearly nonsharp and they conjecture sharp estimates. The aim of the present paper is to give practically sharp estimates in the case of real analytic metrics and in the case of $C^{\infty}$-surfaces. We prove:

Theorem 1.3. Let $(M, g)$ be a closed real analytic Riemannian manifold. Let $\varphi_{\lambda}$ be as above. Let $B \subseteq M$ be an arbitrary metric ball of radius $R$, and let $\Omega_{\lambda}$ be a connected component of $\left\{\varphi_{\lambda} \neq 0\right\} \cap B$. If $\Omega_{\lambda} \cap \frac{1}{2} B \neq \emptyset$ then

$$
\frac{\left|\Omega_{\lambda}\right|}{|B|} \geq \frac{C_{2}}{(\sqrt{\lambda})^{2 n-2} R^{\prime}(\log \lambda)^{n-1}},
$$

where $R^{\prime}=\max \{R, 1 / \sqrt{\lambda}\}$.

In particular, for $R<1 / \sqrt{\lambda}$, we have

$$
\left|\Omega_{\lambda}\right| /|B| \geq C_{2}(\sqrt{\lambda})^{-(n-1)}(\log \lambda)^{-(n-1)} .
$$

To better understand Theorem 1.3 consider a harmonic function $\varphi$ defined in the ball of radius $2, B_{2} \subseteq \mathbb{R}^{n}$. Assume $\varphi(0)=0$, and suppose also that its growth $\beta$ is given by

$$
\beta:=\log \frac{\sup _{B_{1}}|\varphi|}{\sup _{B_{1 / 2}}|\varphi|} .
$$

We consider the positive and negative components of $\varphi$ in the unit ball $B_{1}$. Take one positive component $\Omega$ of $\{\varphi>0\} \cap B_{1}$. We show that if $\Omega \cap B_{1 / 2} \neq \emptyset$ then

$$
\operatorname{Vol}(\Omega) \geq \frac{C}{\beta^{n-1}} .
$$

In fact we prove a similar estimate for a small perturbation of harmonic functions, and use a well known scaling argument to pass to the estimate for eigenfunctions on the wavelegth $(1 / \sqrt{\lambda})$ scale.

One of our main motivation to prove the estimate (1.5) besides the interest raised by Donnelly and Fefferman is the following result in [NPS05]: 
Theorem 1.6. Under the assumptions above in dimension $n=2$

$$
\operatorname{Vol}(\varphi>0) \geq \frac{C}{\log \beta} .
$$

Theorem 1.6 was proved by using complex-analysis methods, and we hope that estimate (1.5) on each positive component separately may lead to a real analysis proof of Theorem 1.6.

We show by a series of examples on the $n$-dimensional round sphere $\mathbb{S}^{n}$, that estimate (1.4) is sharp up to the $(\log \lambda)^{n-1}$ factor:

Theorem 1.7. Consider the standard round sphere $\mathbb{S}^{n}$. For every eigenvalue $\lambda$ and $R<1 / \sqrt{\lambda}$ there exists an eigenfunction $\varphi_{\lambda}$ on $\mathbb{S}^{n}$, a nodal domain $\mathcal{A}_{\lambda}$ and a ball $B$ of radius $R$ such that

$$
\frac{\left|\mathcal{A}_{\lambda} \cap B\right|}{|B|} \leq \frac{C_{3}(n)}{(\sqrt{\lambda})^{n-1}},
$$

and $\mathcal{A}_{\lambda} \cap \frac{1}{2} B \neq \emptyset$.

For $R \sim 1$, we have

$$
\left|\Omega_{\lambda}\right| /|B| \geq C_{2}(\sqrt{\lambda})^{-(2 n-2)}(\log \lambda)^{-(n-1)} .
$$

An example (see Section 7.2) on a flat torus suggests that the power $2 n-2$ in (1.8) could be improved to $n-1$. However, we believe that the large ball behavior should depend on the dynamics of the manifold, and it would be interesting to prove a result in this direction.

We also consider in this paper $C^{\infty}$-surfaces. In this case, we use the methods from [NPS05] in order to reduce the analysis to the case of flat metrics. Then, we can apply complex analysis. We prove

Theorem 1.9. Let $(\Sigma, g)$ be a closed $C^{\infty}$-Riemannian surface. Then

$$
\frac{\left|\Omega_{\lambda}\right|}{|B|} \geq \frac{C_{4}}{\lambda R^{\prime}(\log \lambda)^{1 / 2}},
$$

where $\lambda, \varphi_{\lambda}, B, R, R^{\prime}, \Omega_{\lambda}$ are as in Theorem 1 .3.

Remark. Comparing the last theorem with Theorem 1.3 in the case $n=2$, we see that we gain a $(\log \lambda)^{1 / 2}$ factor. This is due to complex analysis methods. 
One can interpret Theorem 1.9 as an estimate on the size of the so called "avoided crossings" discussed in the physics literature ([MSG03]). Roughly speaking, two nodal lines cannot approach each other too much for a long period of time. Theorem 1.9 shows that two nodal lines cannot approach each other much less than a distance of $1 / \lambda$ (interestingly, no square root here) along a line of length $\geq C / \sqrt{\lambda}$. On the other hand, we showed in Man08 that two adjacent nodal lines cannot stay much closer than $C / \sqrt{\lambda}$ at all times.

\subsection{Methods of Proof}

The main tool which we use in the proof of Theorem 1.3 is a generalization of Hadamard's 3-circles theorem due to Nadirashvili. This lets us eliminate difficult Carleman type estimates which were used in [DF90]. In more details we exploit the following three properties of eigenfunctions:

"Reduction" to Harmonic Functions. We follow the principle that on balls of small radius with respect to the wavelength $1 / \sqrt{\lambda}$ a $\lambda$-eigenfunction is almost harmonic. This principle was developed in [DF88, [DF90], Nad91] and [NPS05]. After rescaling an eigenfunction $\varphi_{\lambda}$ in a ball of radius $\sim 1 / \sqrt{\lambda}$ to the unit ball $B_{1}$, one arrives at a solution $\varphi$ of a second order self adjoint elliptic operator $L$ in the unit ball $B_{1} \subseteq \mathbb{R}^{n}$, where $L$ has coefficients bounded independently of $\lambda . \varphi$ is close to a harmonic function in a sense to be clarified below. Moreover, the growth (defined below) of $\varphi$ in the unit ball is bounded in terms of $\lambda$ ([DF88]).

Rapid Growth in Narrow Domains. If a harmonic function $\varphi$ vanishes on the boundary of a domain which is long and narrow $\varphi$ must grow exponentially fast along the direction in which the domain is long. A corresponding property is true for eigenfunctions on any $C^{\infty}$-manifold.

This was extensively developed and investigated by Landis ([Lan63]) for a certain class of solutions of second order elliptic equations. The version we found in [Lan63] cannot be directly applied to eigenfunctions. A version for eigenfunctions but with slightly weaker estimates than in the present paper was proved in ([DF90]).

In Section 3 we formulate a sharp version of this property which can be applied for eigenfunctions. We prove it in Section 5. The proof combines the ideas from [Lan63] and [DF90]. We replace some arguments from [DF90] by more elementary ones. 
Nadirashvili's-Hadamard's 3-Circles Theorem. This is a "propagation of smallness" principle: Let $\varphi$ be a harmonic function in the unit ball, and suppose $|\varphi| \leq 1$. If a harmonic function $\varphi$ is small on a subset $E \subseteq B_{r}$, where $r<1$ and $|E| /\left|B_{r}\right|$ is large then $|\varphi|$ can be estimated from above in any concentric ball $B_{R}$ containing $B_{r}$. When $E$ is a ball centered at 0 , this reduces to the classical Hadamard's 3-Circles Theorem. Nadirashvili replaced the innermost circle by an arbitrary measurable subset $E$. When this principle is adapted to eigenfunctions, we are restricted to consider real analytic metrics. The sharp estimate in the generalized Hadamard Theorem is the main source from which we get the improvement in Theorem 1.3 relative to Theorem 1.2 ,

\subsection{Organization of the paper}

In Section 2 we rescale the problem on balls of small radius compared with the wavelength to a problem on the unit ball in $\mathbb{R}^{n}$. Thus, we arrive to consider a problem on almost harmonic functions whose growth is controlled. In Section 3 we explain Rapid Growth in Narrow Domains and Propagation of Smallness. In Section 4 we prove Theorem 1.3. In Section 5 we prove Rapid Growth in Narrow Domains. In Section [6 we consider the case of smooth surfaces. We give a new estimate for harmonic functions in dimension two, and show how Theorem 1.9 follows. In Section 7 we give two examples. The first one is a sequence of spherical harmonics which demonstrate that Theorem 1.3 is sharp up to a logarithmic factor for balls of radius smaller than the wavelength. The second one is a sequence of eigenfunctions on the standard flat torus concerning the sharp bound in Theorem 1.3 for balls of radius $\sim 1$.

Acknowledgements: I would like to heartily thank Kolya Nadirashvili for indicating to me that the Generalized Hadamard Theorem may be fruitful. I owe many thanks to Leonid Polterovich and Misha Sodin for their continuous encouragement and helpful discussions. I am grateful to Alexander Eremenko for removing the extra log factor in our estimate in dimension two. Finally, I would like to thank Alexander Borichev and Dima Jakobson for fruitful discussions. This paper was written in the IHES, MPIM-Bonn while the author was an EPDI fellow, and the CRM, Montreal. The support of the EPDI, IHES, MPIM-Bonn and the CRM is gratefully acknowledged. 


\section{Passing to the Wavelength Scale}

Consider an eigenfunction $\varphi_{\lambda}$ on a ball $B \subseteq M$ of radius small compared to the wavelength. Namely, consider $\varphi_{\lambda}$ as a function defined on a Euclidean ball of radius $\sqrt{\varepsilon / \lambda}$ contained in $\mathbb{R}^{n}$ as a coordinate neighbourhood. Now, rescale $\varphi_{\lambda}$ to a function $\varphi$ on the unit ball $B_{1} \subseteq \mathbb{R}^{n}$. $\varphi$ satisfies

$$
L \varphi=0,
$$

where $L$ is a second order elliptic operator with $C^{\infty}$ coefficients. Equation (2.1) with real analytic coefficients will be denoted by (2.1.RA). $L$ is of the form

$$
L u:=-\partial_{i}\left(a^{i j} \partial_{j} u\right)-\varepsilon q u .
$$

$a^{i j}$ is symmetric and satisfies ellipticity bounds:

$$
\kappa_{1}|\xi|^{2} \leq a^{i j} \xi_{i} \xi_{j} \leq \kappa_{2}|\xi|^{2} .
$$

$a^{i j}, q$ are bounded:

$$
\left\|a^{i j}\right\|_{C^{1}\left(\overline{B_{1}}\right)} \leq K,|q| \leq K,
$$

and we will assume $\varepsilon<\varepsilon_{0}$, and $\varepsilon_{0}$ is small.

It is a fundamental fact due to Donnelly and Fefferman that the eigenvalue $\lambda$ controls the growth of the eigenfunction $\varphi_{\lambda}$ and hence also of $\varphi$ : For a Euclidean ball $B \subseteq B_{1}$, we define the $r$-growth exponent as:

$$
\beta_{r}(\varphi ; B):=\log \frac{\sup _{B}|\varphi|}{\sup _{r B}|\varphi|} \quad, \quad \beta_{r}(\varphi):=\sup _{B \subseteq B_{1}} \beta_{r}(\varphi ; B) .
$$

Donnelly and Fefferman proved ([DF88]) that for the rescaled eigenfunction $\varphi$ we have

$$
\frac{\beta_{r}(\varphi)}{\log (1 / r)} \leq C \sqrt{\lambda}
$$

\section{Rapid Growth and Propagation of Small- ness}

In this section we give precise formulations of the properties mentioned in the introduction. We use them in Section 4 , 
- Rapid Growth in Narrow Domains: This property tells that if a solution $\varphi$ of (2.1) has a deep and narrow positivity component $\Omega$, then $\varphi$ grows rapidly in $\Omega$. A first version of it is given by

Theorem 3.1. Let $\varphi$ satisfy (2.1). Let $\Omega$ be a connected component of $\{\varphi>0\}$ which intersects $B_{1 / 2}$. Let $\eta>0$ be small enough. If

$$
\frac{|\Omega|}{\left|B_{1}\right|} \leq \eta^{n-1}
$$

then

$$
\frac{\sup _{\Omega} \varphi}{\sup _{\Omega \cap B_{1 / 2}} \varphi} \geq \mathrm{e}^{C_{1} / \eta}
$$

We remark that $\eta$ can be considered as a bound on the cross sections of $\Omega$. We will use an iterated version of the above property:

Theorem 3.2. Let $\varphi$ satisfy (2.1). Let $0<r_{0} \leq 1 / 2$. Let $\Omega$ be a connected component of $\{\varphi>0\}$ which intersects $B_{r_{0}}$. Let $\eta>0$ be small enough. If $\left|\Omega \cap B_{r}\right| /\left|B_{r}\right| \leq \eta^{n-1}$ for all $r_{0}<r<1$, then

$$
\frac{\sup _{\Omega} \varphi}{\sup _{\Omega \cap B_{r_{0}}} \varphi} \geq\left(\frac{1}{r_{0}}\right)^{C_{1} / \eta}
$$

\section{- Nadirashvili's-Hadamard's 3-Circles Theorem:}

Theorem 3.3 ([Nad76]). Let $\varphi$ satisfy [2.1.RA), Let $E \subseteq B_{R}$, where $R<1$.

$$
\text { If } \frac{\sup _{E}|\varphi|}{\sup _{B_{1}}|\varphi|} \leq\left(\frac{|E|}{\left|B_{1}\right|}\right)^{\gamma / n} \text {, then } \frac{\sup _{B_{R}}|\varphi|}{\sup _{B_{1}}|\varphi|} \leq\left(c_{0} R\right)^{c_{1} \gamma}
$$

whenever $\gamma>\gamma_{0}$, and where $\gamma_{0}, c_{0}, c_{1}$ depend on $\kappa_{1}, \kappa_{2}, K, \varepsilon_{0}, n$.

When $L$ is the Euclidean Laplacian, $n=2$ and $E$ is a ball centered at 0 we get the classical Hadamard Theorem which says that the maximal function $\max _{B_{r}}|\varphi|$ is a logarithmic convex function of $\log r$. When $E=B_{R}$ and $n>2$, this theorem was proved by Gerasimov in Ger66.

Observation. Theorem 3.3 is equivalent to

$$
\sup _{B_{1}}|\varphi| \leq \sup _{E}|\varphi| \cdot\left(\frac{\left|B_{1}\right|}{|E|}\right)^{C \beta_{R}\left(\varphi ; B_{1}\right) / \log (1 / R)}
$$


for all $R$ small enough and where $E \subseteq B_{R}$. One should compare (3.4) with inequality (D) in [DF90, p. 636].

Remark. The proof in Nad76] is for harmonic functions. When one goes through the proof, one sees that the only point where the harmonicity of $\varphi$ is used is an interior elliptic regularity estimate which is true for solutions of any second order elliptic operator with real analytic coefficients (See [Hör64, Theorem 7.5.1]).

\section{Proof of Theorem 1.3}

From Section 2 it follows that on balls of radius $R<\sqrt{\varepsilon_{0} / \lambda}$ Theorem 1.3 is equivalent to Theorem 1.3 below. In section 4.1 we complete the argument for larger balls.

Recall the definition (2.5) of $\beta_{r}$. We set

$$
\beta_{r}^{\prime}(\varphi ; B):=\max \left\{\beta_{r}(\varphi ; B), 3\right\}, \quad \beta_{r}^{\prime}(\varphi):=\max \left(\beta_{r}(\varphi), 3\right) .
$$

Theorem 1.3'. Let $\varphi$ satisfy (2.1.RA). Let $\Omega$ be a connected component of $\{\varphi \neq 0\}$. Suppose $\Omega \cap B_{1 / 2} \neq \emptyset$. Then,

$$
\frac{|\Omega|}{\left|B_{1}\right|} \geq \frac{C_{1}}{\left(\beta_{\rho_{0}}^{\prime}(\varphi) \log \beta_{\rho_{0}}^{\prime}(\varphi)\right)^{n-1}}
$$

where $0<\rho_{0}<1$ depends only on $K, \kappa_{1}, \kappa_{2}, \varepsilon_{0}$ and $n$.

Theorem 1.3 immediately follows from

Theorem 1.3". Let $\varphi$ satisfy (2.1.RA). Suppose $\varphi(0)>0$. Let $\Omega$ be the connected component of $\{\varphi>0\}$ which contains 0 . Then

$$
\frac{|\Omega|}{\left|B_{1}\right|} \geq\left(\frac{C_{1} \varepsilon}{\beta_{\rho_{0}}^{\prime}\left(\varphi ; B_{1}\right)}\right)^{(n-1) /(1-\varepsilon)}
$$

for all $0<\varepsilon<1 / 2$, and where $0<\rho_{0}<1$ depends only on $K, \kappa_{1}, \kappa_{2}, \varepsilon_{0}, n$.

Theorem 1.3]" implies Theorem [1.3]'. One may use an affine transformation in order to move the center of the ball to $\Omega \cap B_{1 / 2}$. Also, one can check that

$$
\sup _{0<\varepsilon<1 / 2}(\varepsilon / A)^{1 /(1-\varepsilon)} \geq C / A \log A \text {. }
$$


Indeed, with $\varepsilon=1 / \log A$, one has

$$
\left(\frac{\varepsilon}{A}\right)^{1 /(1-\varepsilon)} \geq \frac{\varepsilon}{A}\left(\frac{\varepsilon}{A}\right)^{2 \varepsilon}=\frac{\mathrm{e}^{2 \varepsilon \log \varepsilon-2}}{A \log A} \geq \frac{\mathrm{e}^{-3}}{A \log A} .
$$

We now give the heart of the argument which proves Theorem 1.3'. It is close to the argument in [DF90]. The main new tool we introduce in the argument is the propagation of smallness principle in the form of Nadirashvili'sHadamard's 3-circles Theorem.

Idea of Proof of Theorem 1.3]". If $|\varphi| \leq 1$ in the ball $B_{1}$ and $|\Omega|$ is very small, then by Rapid Growth in Narrow Domains $\varphi$ should be very small on $E_{1 / 2}:=\left|\Omega \cap B_{1 / 2}\right|$. Then by the propagation of smallness from $E_{1 / 2}$ to $B_{1 / 2}$, we know that $|\varphi|$ must be small also on the ball $B_{1 / 2}$, but if it is too small we will get that the growth of $\varphi$ on $B_{1}$ is bigger than $\beta_{1 / 2}\left(\varphi ; B_{1}\right)$. The argument above has to be modified a little bit, since we can apply propagation of smallness only if we know that the relative size of $E_{1 / 2}$ in $B_{1 / 2}$ is large. All one has to do is to replace $E_{1 / 2}$ by $E_{r}$ for a suitable $r<1 / 2$.

We now give the complete proof.

Proof of Theorem 1.3]". We may assume

$$
|\Omega| /\left|B_{1}\right|<\left(\varepsilon / \gamma_{1}\right)^{(n-1) /(1-\varepsilon)},
$$

where $\gamma_{1}$ is a large enough constant. Otherwise, the theorem becomes trivial. Let $c_{0}>1$ be as in Theorem 3.3, and let $\rho_{0}=1 /\left(2 c_{0}\right)$. Define

$$
r_{0}:=\sup \left\{r: \frac{\left|\Omega \cap B_{r}\right|}{\left|B_{r}\right|} \geq \frac{1}{\rho_{0}^{n}}\left(\frac{|\Omega|}{\left|B_{1}\right|}\right)^{1-\varepsilon}\right\} .
$$

Let $E:=\Omega \cap B_{r_{0}}$.

\section{Claim 4.3.}

$$
r_{0}<\rho_{0}\left(|E| /\left|B_{1}\right|\right)^{\varepsilon / n}
$$

Proof of Claim. By definition,

$$
\left(r_{0} / \rho_{0}\right)^{n} \leq\left(|\Omega| /\left|B_{1}\right|\right)^{\varepsilon}(|E| /|\Omega|) \leq\left(|\Omega| /\left|B_{1}\right|\right)^{\varepsilon}(|E| /|\Omega|)^{\varepsilon} .
$$


In particular,

$$
r_{0} \leq \min \left\{\rho_{0},\left(|E| /\left|B_{1}\right|\right)^{\varepsilon / n}\right\} .
$$

Theorem 3.2 and Inequality (4.4) give together

$$
\begin{array}{r}
\log \frac{\sup _{E}|\varphi|}{\sup _{B_{1}}|\varphi|} \leq \log \frac{\sup _{E}|\varphi|}{\sup _{\Omega}|\varphi|} \leq C_{1}\left(\log r_{0}\right) \rho_{0}^{n /(n-1)} /\left(|\Omega| /\left|B_{1}\right|\right)^{(1-\varepsilon) /(n-1)} \leq \\
C_{2} \varepsilon\left(\log \left(|E| /\left|B_{1}\right|\right)^{1 / n}\right) /\left(|\Omega| /\left|B_{1}\right|\right)^{(1-\varepsilon) /(n-1)}
\end{array}
$$

Due to assumption (4.1) and to $E \subseteq B_{\rho_{0}}$ we can apply Theorem 3.3.

$$
-\beta_{\rho_{0}}\left(\varphi ; B_{1}\right)=\log \frac{\sup _{B_{\rho_{0}}}|\varphi|}{\sup _{B_{1}}|\varphi|} \leq C_{3} \varepsilon\left(\log c_{0} \rho_{0}\right) /\left(|\Omega| /\left|B_{1}\right|\right)^{(1-\varepsilon) /(n-1)} .
$$

The last inequality can be rewritten as

$$
\frac{|\Omega|}{\left|B_{1}\right|} \geq\left(\frac{\varepsilon C_{4}}{\beta_{\rho_{0}}\left(\varphi ; B_{1}\right)}\right)^{(n-1) /(1-\varepsilon)} .
$$

\subsection{Handling Large Balls}

So far, we proved Theorem 1.3 for small balls. We now treat the case of balls $B$ whose radius radius $R>\sqrt{\varepsilon_{0} / \lambda}$. There are two cases:

1) $\Omega_{\lambda}=\mathcal{A}_{\lambda} \subseteq B$. By the Faber-Krahn Inequality, we know that $\left|\Omega_{\lambda}\right| \geq$ $C_{2} /(\sqrt{\lambda})^{n}$. Hence,

$$
\frac{\left|\Omega_{\lambda}\right|}{|B|} \geq \frac{C_{3}}{(R \sqrt{\lambda})^{n}}
$$

which gives the estimate in Theorem 1.3.

2) $\Omega_{\lambda}$ touches $\partial B$. We decompose $B \backslash \frac{1}{2} B$ into spherical layers, each of width $\sqrt{\varepsilon_{0} / \lambda}$. In each spherical layer we can find a ball $B^{\prime}$ of radius $\left(\sqrt{\varepsilon_{0} / \lambda}\right) / 2$ such that $\Omega_{\lambda}$ cuts $\frac{1}{2} B^{\prime}$. The number of such layers is $\sim$ $R \sqrt{\lambda / \varepsilon_{0}}$. By Theorem 1.3 for small balls the total volume of $\Omega_{\lambda}$ is

$$
\left|\Omega_{\lambda}\right| \geq \sum_{B^{\prime}}\left|\Omega_{\lambda} \cap B^{\prime}\right| \geq C_{5} R \sqrt{\lambda / \varepsilon_{0}}\left|B^{\prime}\right| /(\sqrt{\lambda} \log \lambda)^{n-1} .
$$


The last inequality gives

$$
\frac{\left|\Omega_{\lambda}\right|}{|B|} \geq \frac{C_{6}}{(R \sqrt{\lambda})^{n-1}(\sqrt{\lambda} \log \lambda)^{n-1}} .
$$

This completes the proof of Theorem 1.3.

\section{Rapid Growth in Narrow Domains}

In this section we prove Theorems 3.1 and 3.2. They follow from the classical growth Lemma: Let $\varphi$ satisfy (2.1). Let $B_{R}^{y}=B(y, R) \subseteq B_{1}$. Suppose $\varphi(y)>0$, and let $\Omega_{y}$ be the connected component of $\{\varphi>0\} \cap B_{R}^{y}$ which contains $y$. The Growth Lemma is

Lemma 5.1 ([Lan63, Lemma 3.1], [DF90, p. 651]). For all $A>1$ there exists $\gamma(A)>0$ such that if

$$
\frac{\left|\Omega_{y}\right|}{\left|B_{R}^{y}\right|} \leq \gamma(A)
$$

then

$$
\frac{\sup _{\Omega_{y}}|\varphi|}{\sup _{\Omega_{y} \cap B_{R / 2}^{y}}|\varphi|} \geq A .
$$

In particular, $\gamma$ does not depend on $R$, neither on $y$.

We give a proof of this lemma in Section 5.1. As a corollary we obtain Theorem 3.1.

Proof of Theorem 3.1. We notice that $\Omega$ touches $\partial B_{1}$. Otherwise, since $\varepsilon<$ $\varepsilon_{0}$ the maximum principle ([GT83, Cor. 3.8]) tells us that $\varphi$ is identically 0 in $\Omega$, which contradicts the definition of $\Omega$.

We decompose $B_{1} \backslash B_{1 / 2}$ into $N$ equally distanced spherical layers, where $N$ will be chosen below. Let $t_{k}=(1 / 2+k /(2 N)), k=0 \ldots N$. Let $A_{0}=B_{1 / 2}$, and $A_{k}=B_{t_{k}} \backslash B_{t_{k-1}}$ for $k=1 \ldots N$. Set $\widetilde{A_{0}}=A_{0} \cup A_{1}, \widetilde{A_{N}}=A_{N-1} \cup A_{N}$, and

$$
\widetilde{A_{k}}=A_{k-1} \cup A_{k} \cup A_{k+1}
$$

for $1 \leq k \leq N-1$. There exist $\geq N / 2$ values of $1 \leq k \leq N$ for which

$$
\left|\Omega \cap \widetilde{A_{k}}\right| \leq 6|\Omega| / N
$$


Let $l$ be any one of these values. Let $y \in \Omega \cap A_{l}$, and let $R=1 /(2 N)$. Consider the ball $B_{R}^{y}=B(y, R) . B_{R}^{y} \subseteq \widetilde{A}_{l}$, and we check that

$$
\frac{\left|\Omega \cap B_{R}^{y}\right|}{\left|B_{R}^{y}\right|} \leq \frac{\left|\Omega \cap \widetilde{A}_{l}\right|}{\left|B_{1}\right|(1 /(2 N))^{n}} \leq \frac{6|\Omega|(2 N)^{n}}{N\left|B_{1}\right|} \leq 12(2 \eta N)^{n-1} .
$$

Set $A=10 \mathrm{e} / 9$ and take $N=\left\lfloor\left((\gamma(A) / 12)^{1 /(n-1)} /(2 \eta)\right)\right\rfloor$. Inequality (5.2) and Lemma 5.1 applied in $B_{R}^{y}$ show that

$$
\sup _{\Omega \cap \widetilde{A}_{l}} \varphi \geq \sup _{\Omega \cap B_{R}^{y}} \varphi \geq A \varphi(y) .
$$

Since this is true for all $y \in \Omega \cap A_{l}$, we get

$$
\sup _{\Omega \cap B_{t_{l+1}}} \varphi \geq \sup _{\Omega \cap \widetilde{A}_{l}} \varphi \geq A \sup _{\Omega \cap A_{l}} \varphi .
$$

Now we apply the following maximum principle:

Theorem 5.4 ([GT83, Corollary 3.8]). Let $\varphi$ satisfy (2.1). Then

$$
\sup _{\Omega \cap A_{k}} \varphi \geq 0.9 \sup _{\Omega \cap B_{t_{k}}} \varphi
$$

whenever $\varepsilon<\varepsilon_{0}$.

Hence, from (5.3) we obtain

$$
\sup _{\Omega \cap B_{t_{l+1}}} \varphi \geq 0.9 A \sup _{\Omega \cap B_{t_{l}}} \varphi=\mathrm{e} \sup _{\Omega \cap B_{t_{l}}} \varphi .
$$

And since this is true for $\geq N / 2$ values of $k$ we finally have

$$
\frac{\sup _{\Omega} \varphi}{\sup _{\Omega \cap B_{1 / 2}} \varphi} \geq \mathrm{e}^{N / 2} \geq \mathrm{e}^{C_{1} / \eta}
$$

An iteration of Theorem 3.1 gives Theorem 3.2,

Proof of Theorem 3.9. Let $N$ be a positive integer for which $(1 / 2)^{N+1}<r_{0} \leq$ $(1 / 2)^{N} . N=\left\lfloor\log \left(1 / r_{0}\right) / \log 2\right\rfloor$. Set $t_{k}=(1 / 2)^{k}$. It follows by scaling from Theorem 3.1 that

$$
\frac{\sup _{\Omega \cap B_{t_{k}}}|\varphi|}{\sup _{\Omega \cap B_{t_{k+1}}}|\varphi|} \geq \mathrm{e}^{C_{1} / \eta}
$$


for all $0 \leq k \leq N-1$. The point is that the bounds (2.3)-(2.4) on the operator $L$ remain true after rescaling. Hence,

$$
\frac{\sup _{\Omega}|\varphi|}{\sup _{\Omega \cap B_{r_{0}}}|\varphi|} \geq \frac{\sup _{\Omega}|\varphi|}{\sup _{\Omega \cap B_{t_{N}}}|\varphi|} \geq \mathrm{e}^{C_{1} N / \eta} \geq \mathrm{e}^{C_{1} \log \left(1 / r_{0}\right) /(2 \eta \log 2)}=\left(1 / r_{0}\right)^{C_{2} / \eta}
$$

\subsection{Proof of the Growth Lemma}

Its proof is based on ideas from the proof in [DF90], where we replaced several arguments by more elementary ones.

Proof of Lemma 5.1. Let $g(t)$ be a smooth function defined on $\mathbb{R}$ with the following properties

- $g(t)=0$ for $t \leq 1$,

- $g(t)=t-2$ for $t \geq 3$,

- $g^{\prime \prime}(t) \geq 0$.

Let $\delta>0$ be small and let $g_{\delta}(t)=\delta g(t / \delta)$. Let $\varphi_{\delta}=\left(g_{\delta} \circ \varphi\right) \cdot \chi_{\Omega_{y}}$, where $\chi_{\Omega_{y}}$ is the characteristic function of $\Omega_{y} \cdot \varphi_{\delta}$ is a smooth function in $B_{R}^{y}$ with compact support in $\Omega_{y}$. We notice that $0 \leq \varphi \chi_{\Omega_{y}}-\varphi_{\delta} \leq 2 \delta$. In particular, $\varphi_{\delta} \rightarrow \varphi \chi_{\Omega_{y}}$ uniformly as $\delta \rightarrow 0$. We now calculate $L \varphi_{\delta}$ :

$$
\begin{aligned}
& L \varphi_{\delta}=\chi_{\Omega_{y}} \varepsilon q \varphi \cdot\left(g_{\delta}^{\prime} \circ \varphi\right)-\varepsilon q \varphi_{\delta}-\chi_{\Omega_{y}} a^{i j} \partial_{i} \varphi \partial_{j} \varphi \cdot\left(g_{\delta}^{\prime \prime} \circ \varphi\right) \\
& \leq \chi_{\Omega_{y}} \varepsilon q \varphi \cdot\left(g_{\delta}^{\prime} \circ \varphi\right)-\varepsilon q \varphi_{\delta}
\end{aligned}
$$

The last inequality is true due to the fact that $a^{i j}$ is positive definite and $g$ is convex. Let us denote the last expression in (5.5) by $f$.

\section{Lemma 5.6.}

$$
\|f\|_{L^{n}\left(B_{R}^{y}\right)} \leq 3 \varepsilon_{0} \delta K\left|\Omega_{y}\right|^{1 / n}
$$

We postpone the proof of this lemma to the end of this section. Recall the following local maximum principle: 
Theorem 5.7 ([GT83, Theorem 9.20]). Suppose Lu $\leq f$ in $B_{R}^{y} \subseteq B_{1}$. Then,

$$
\sup _{B_{R / 2}^{y}} u \leq \frac{C_{1}}{\left|B_{R}^{y}\right|} \int_{B_{R}^{y}} u^{+} d x+C_{2}\|f\|_{L^{n}\left(B_{R}^{y}\right)},
$$

where $C_{1}, C_{2}$ depend only on $\kappa_{1}, \kappa_{2}$ and $K$.

Applying the local maximum principle to $\varphi_{\delta}$ gives

$$
\sup _{B_{R / 2}^{y}} \varphi_{\delta} \leq \frac{C_{1}}{\left|B_{R}^{y}\right|} \int_{B_{R}^{y}} \varphi_{\delta} d x+3 C_{2} \delta \varepsilon_{0} K\left|\Omega_{y}\right|^{1 / n} .
$$

Letting $\delta \rightarrow 0$ we obtain that

$$
\sup _{\Omega_{y} \cap B_{R / 2}^{y}} \varphi \leq \frac{C_{1}}{\left|B_{R}^{y}\right|} \int_{\Omega_{y}} \varphi d x \leq \frac{C_{1}\left|\Omega_{y}\right|}{\left|B_{R}^{y}\right|} \sup _{\Omega_{y}} \varphi .
$$

Thus, we may take $\gamma(A)=1 /\left(C_{1} A\right)$.

To complete the proof of the Growth Lemma 5.1 it remains to prove Lemma 5.6.

Proof of Lemma 5.6. We notice that $t-2 \delta \leq g_{\delta}(t) \leq t$ and $0 \leq g_{\delta}^{\prime}(t) \leq 1$. Hence, $f \leq \varepsilon q \varphi \chi_{\Omega_{y}}-\varepsilon q(\varphi-2 \delta) \chi_{\Omega_{y}}=2 \varepsilon \delta q \chi_{\Omega_{y}}$. When $\varphi \geq 3 \delta, f=2 \varepsilon \delta q \chi_{\Omega_{y}}$, and when $\varphi \leq 3 \delta, f \geq-\varepsilon q \varphi \chi_{\Omega_{y}} \geq-3 \varepsilon q \delta \chi_{\Omega_{y}}$. We have shown that $|f| \leq$ $3 \varepsilon_{0} \delta q \chi_{\Omega_{y}}$. Integration gives

$$
\|f\|_{L^{n}\left(B_{R}^{y}\right)} \leq 3 \varepsilon_{0} \delta K\left|\Omega_{y}\right|^{1 / n} .
$$

\section{Dimension Two - $C^{\infty}$ case}

In dimension two we can use the techniques in [NPS05 in order to reduce the analysis to the case of harmonic functions. 


\subsection{An estimate for harmonic functions}

Let $\varphi$ be a harmonic function in the unit ball $B_{1} \subseteq \mathbb{R}^{2}$. Let $\Omega$ be a connected component of $\{\varphi \neq 0\}$. Suppose $\Omega \cap B_{1 / 2} \neq \emptyset$. Theorem 1.3 shows

$$
|\Omega| /\left|B_{1}\right|>C / \beta_{\rho_{0}}^{\prime}(\varphi) \log \beta_{\rho_{0}}^{\prime}(\varphi)
$$

for some $0<\rho_{0}<1$. We will show that in fact,

\section{Theorem 6.1.}

$$
|\Omega| /\left|B_{1}\right| \geq C / \beta_{1 / 2}^{\prime}\left(\varphi ; B_{1}\right)
$$

In order to see this we define $h=\varphi$ in $\Omega$ and $h=0$ elsewhere in $B_{1}$. $h$ is a subharmonic function. The key is the following proposition:

Theorem 6.2 (Eremenko).

$$
\beta_{3 / 4}\left(h ; B_{1}\right) \leq C_{1} \beta_{1 / 2}\left(\varphi ; B_{1}\right)+C_{2} .
$$

Remark. Nadirashvili's-Hadamard's Theorem is equivalent to a related inequality with $C_{1}=C_{1}(\Omega)$. Namely,

$$
\frac{\beta_{r}\left(h ; B_{1}\right)}{\log \left(1 /\left(\left|\Omega \cap B_{r}\right| /\left|B_{1}\right|\right)^{1 / n}\right)} \leq C_{1} \frac{\beta_{r}\left(\varphi ; B_{1}\right)}{\log 1 /\left(C_{2} r\right)} .
$$

The important point in Theorem 6.2 is that by allowing a slightly bigger $r$ in the nominator of the LHS of (6.3) we obtain a constant $C_{1}$ which is independent of $\Omega$.

Proof of Theorem 6.2. The proof is based on the harmonic majorant principle and on the Beurling-Nevannlina projection theorem. For a function $u$ defined in $B_{1}$, let $M_{u}(r):=\max _{|z| \leq r}|u|$. We normalize $\varphi$ so that $M_{\varphi}(1)=1$. It is well known ([Hör94]) that $M_{h}(r)$ is a convex function of $\log r$, that is, $t M_{h}^{\prime}(t)$ is a monotonically increasing function of $t$. Thus, we have for all $1 / 2 \leq t \leq 2 / 3$

$$
\begin{gathered}
\mathrm{e}^{-\beta_{3 / 4}\left(h ; B_{1}\right)} \geq M_{h}(3 / 4)-M_{h}(t)=\int_{t}^{3 / 4} \frac{r M_{h}^{\prime}(r)}{r} d r \geq \\
t M_{h}^{\prime}(t) 4(3 / 4-t) / 3 \geq t M_{h}^{\prime}(t) / 9 \geq M_{h}^{\prime}(t) / 18 .
\end{gathered}
$$


Hence, $M_{h}^{\prime}(t)<20 \mathrm{e}^{-\beta_{3 / 4}\left(h ; B_{1}\right)}$ for all $1 / 2 \leq t \leq 2 / 3$.

Now, let $z_{t}$ be a point where $h\left(z_{t}\right)=M_{h}(t)$. Then,

$$
0 \leq \frac{\partial h}{\partial r}\left(z_{t}\right) \leq M_{h}^{\prime}(t), \quad \frac{\partial h}{\partial \theta}\left(z_{t}\right)=0
$$

Thus, we get that on $z_{t}$

$$
\left|\nabla \varphi\left(z_{t}\right)\right| \leq 20 \mathrm{e}^{-\beta_{3 / 4}\left(h ; B_{1}\right)}
$$

The function $v:=\log |\nabla \varphi|$ is subharmonic in $B_{1}$, and on $z_{t}$,

$$
v\left(z_{t}\right) \leq-\beta_{3 / 4}\left(h ; B_{1}\right)+C_{1} .
$$

Since on $|z| \leq 1,|\varphi| \leq 1$, on the circle $|z|=2 / 3,|\nabla \varphi| \leq C_{2}$. So,

$$
\left.v\right|_{|z|=2 / 3} \leq C_{3} \text {. }
$$

Now we apply the harmonic majorant principle: Let $\omega\left(z, \gamma, B_{2 / 3}\right)$ be the harmonic measure of $\gamma=\left\{z_{t}: 1 / 2 \leq t \leq 2 / 3\right\}$ with respect to $B_{2 / 3}$. $\omega$ is a harmonic function on $B_{2 / 3} \backslash \gamma$, which tends to 1 on $z_{t}$ and to 0 on $|z|=2 / 3$. Thus, in light of (6.4) and (6.5), we get

$$
v \leq\left(-\beta_{3 / 4}\left(h ; B_{1}\right)+C_{1}\right) \omega+C_{3} \leq-\beta_{3 / 4}\left(h ; B_{1}\right) \omega+C_{4} .
$$

Also, since $M_{\varphi}(1 / 2) \geq \mathrm{e}^{-\beta_{1 / 2}\left(\varphi ; B_{1}\right)}$, there exists a point $z_{0},\left|z_{0}\right| \leq 1 / 2$, where

$$
v\left(z_{0}\right) \geq-\beta_{1 / 2}\left(\varphi ; B_{1}\right)+C_{5} .
$$

By the Beurling-Nevannlina Projection Theorem ([Ahl73])

$$
\omega\left(z_{0}, \gamma, D\right) \geq \omega\left(-\left|z_{0}\right| ;[1 / 2,2 / 3], B_{2 / 3}\right)=C_{6} .
$$

Combining (6.8) with (6.6) and (6.7), we obtain

$$
-\beta_{1 / 2}\left(\varphi ; B_{1}\right)+C_{5} \leq v\left(z_{0}\right) \leq-C_{6} \beta_{3 / 4}\left(h ; B_{1}\right)+C_{4} .
$$

Proof of Theorem 6.1. By Theorem 3.1

$$
\beta_{3 / 4}\left(h ; B_{1}\right) \geq C /\left(|\Omega| /\left|B_{1}\right|\right) .
$$

On the other hand, Theorem 6.2 tells us

$$
\beta_{3 / 4}\left(h ; B_{1}\right) \leq C_{1} \beta_{1 / 2}\left(\varphi ; B_{1}\right)+C_{2} .
$$

Combining these two inequalities we get

$$
|\Omega| /\left|B_{1}\right| \geq C_{1} /\left(\beta_{1 / 2}\left(\varphi ; B_{1}\right)+C_{2}\right) \geq C_{3} / \beta_{1 / 2}^{\prime}\left(\varphi ; B_{1}\right) .
$$




\subsection{Proof of Theorem 1.9}

The technique developed in [NPS05] shows that one can use a quasiconformal mapping in order to pass to estimates for harmonic functions.

From Section 2 and by using conformal coordinates we see that Theorem 1.9 is equivalent for small balls to

Theorem 6.9. Let $\Delta \varphi-\varepsilon q \varphi=0$ in $B_{1} \subseteq \mathbb{C}$. Suppose $\varphi(0)>0$ and let $\Omega \subseteq B_{1}$ be the connected component of $\{\varphi>0\}$ which contains 0 . Then,

$$
\frac{|\Omega|}{\left|B_{1}\right|} \geq \frac{C_{3}}{\beta_{1 / 2}^{\prime}(\varphi)\left(\log \beta_{1 / 2}^{\prime}(\varphi)\right)^{1 / 2}} .
$$

Proof. The theorem follows from Theorem 6.1 in exactly the same way which is explained in [NPS05. So, we omit the proof.

Then, for large balls we proceed in the same way as in Section 4.1.

\section{Examples}

\subsection{An Example on $\mathbb{S}^{n}$}

In this section we show that Theorem 1.3 for balls of radius $R<1 / \sqrt{\lambda}$ is sharp up to the $(\log \lambda)^{n-1}$ factor. The example we give will be a sequence of spherical harmonics on the standard sphere $\mathbb{S}^{n}$. Let us denote by $\mathcal{H}_{k}^{n}$ the space of spherical harmonics on $\mathbb{S}^{n}$ of degree $k$.

Proposition 7.1. There exists a sequence $\left(Y_{k}^{n}\right)_{k \geq 1} \in \mathcal{H}_{k}^{n}$ with the following properties:

1. The number of nodal domains of $Y_{k}^{n}$ is $\geq c_{1, n} k^{n}$.

2. There exist $\geq c_{2, n} k^{n-1}$ nodal domains of $Y_{k}^{n}$ which have the north pole on their boundary.

Corollary 7.2. For every eigenvalue $\lambda$ and $r<1 / \sqrt{\lambda}$ there exists an eigenfunction $\varphi_{\lambda}$, a nodal domain $\mathcal{A}_{\lambda}$ and a ball $B$ of radius $r$ such that

$$
\frac{\operatorname{Vol}\left(\mathcal{A}_{\lambda} \cap B\right)}{\operatorname{Vol}(B)} \leq \frac{C_{3}(n)}{(\sqrt{\lambda})^{n-1}},
$$

and $\mathcal{A}_{\lambda} \cap \frac{1}{2} B \neq \emptyset$. 
Proof. $\lambda=k(k+n-1)$ for some integer $k \geq 0$. Let $Y_{k}^{n}$ be as in Proposition [7.1. Let $B$ be a ball of radius $r<1 / k$ centered at the north pole. By Proposition 7.1 there exists a nodal domain $\mathcal{A}_{\lambda}$ for which

$$
\frac{\operatorname{Vol}\left(\mathcal{A}_{\lambda} \cap B\right)}{\operatorname{Vol}(B)} \leq \frac{C_{4}(n)}{k^{n-1}}
$$

The result follows since $\lambda \sim k^{2}$.

We now prove Proposition 7.1. First, we introduce spherical coordinates and we review elementary facts about spherical harmonics.

Lemma 7.3. A point on the sphere $\mathbb{S}^{n}$ is parametrized by $\left(\theta_{1}, \ldots, \theta_{n-1}, \varphi\right)$, where $0<\theta_{l}<\pi, 0 \leq \varphi \leq 2 \pi$, and

$$
\begin{aligned}
& x_{1}=\cos \theta_{1} \\
& \vdots \\
& x_{n-1}=\sin \theta_{1} \ldots \sin \theta_{n-2} \cos \theta_{n-1}, \\
& x_{n}=\sin \theta_{1} \ldots \sin \theta_{n-1} \cos \varphi, \\
& x_{n+1}=\sin \theta_{1} \ldots \sin \theta_{n-1} \sin \varphi .
\end{aligned}
$$

We recall the definition of the zonal spherical harmonics and Legendre Polynomials. Details can be found in chapter 3 of [Gro96]. Consider the natural action of the orthogonal group $O(n+1)$ on $\mathbb{S}^{n}$. It induces a representation of $O(n+1)$ on $\mathcal{H}_{k}^{n}$. The zonal spherical harmonic $Z_{k, p}^{n}$ of degree $k$ with pole $p \in \mathbb{S}^{n}$ is defined as the unique spherical harmonic in $\mathcal{H}_{k}^{n}$, which is fixed by the stabilizer of the point $p$ in $O(n+1)$, and admits the value 1 at $p$. The Legendre polynomial $P_{k}^{n+1}(t)$ is defined to be the polynomial on $[-1,1]$, for which

$$
Z_{k, p_{0}}^{n}\left(\theta_{1}, \ldots, \theta_{n-1}, \phi\right)=P_{k}^{n+1}\left(\cos \theta_{1}\right)
$$

where $p_{0}$ is the north pole. It is easy to see that for any $p \in \mathbb{S}^{n}$

$$
Z_{k, p}^{n}(x)=P_{k}^{n+1}(\langle p, x\rangle)
$$

Lemma 7.4 ([Gro96, Proposition 3.3.7]). $P_{k}^{n}$ is given by

$$
P_{k}^{n}(t)=\alpha_{n}(-1)^{k}\left(1-t^{2}\right)^{-(n-3) / 2} \frac{\partial^{k}}{\partial t^{k}}\left(1-t^{2}\right)^{k+\frac{n-3}{2}},
$$

where $\alpha_{n}$ are some constants which depend on $n$. 
We define also the associated Legendre functions:

$$
E_{k, j}^{n}(t)=\left(1-t^{2}\right)^{j / 2}\left(\partial_{t}^{j} P_{k}^{n}\right)(t) .
$$

The next lemma is an inductive construction of spherical harmonics:

Lemma 7.5 ([Gro96, Lemma 3.5.3]). Given $G \in \mathcal{H}_{j}^{n-1}$, let

$$
H\left(\theta_{1}, \ldots \theta_{n-1}, \varphi\right):=E_{k, j}^{n+1}\left(\cos \theta_{1}\right) G\left(\theta_{2}, \ldots \theta_{n-1}, \varphi\right) .
$$

Then, $H \in \mathcal{H}_{k}^{n}$.

Proof of Proposition 7.1. We prove it by induction on $n$. For $n=1$, we take $Y_{k}^{1}(\varphi)=\sin k \varphi$. Suppose the result is true for $n-1$. Set

$$
H_{k, j}^{n}\left(\theta_{1}, \ldots, \theta_{n-1}, \varphi\right):=E_{k, j}^{n+1}\left(\cos \theta_{1}\right) Y_{j}^{n-1}\left(\theta_{2}, \ldots, \theta_{n-1}, \varphi\right) .
$$

By Lemma $7.5 H_{k, j}^{n} \in \mathcal{H}_{k}^{n}$. From Lemma 7.4 one can see that $E_{k, j}^{n+1}$ has exactly $k-j$ distinct zeroes in the interval $(-1,1)$ it follows that the number of nodal domains of $H_{n}^{k}$ is $\geq c_{1, n-1}(k-j) j^{n-1}$, of which $c_{1, n-1} j^{n-1}$ touch the north pole. We define $Y_{k}^{n}:=H_{k,\lfloor k / 2\rfloor}^{n}$.

\subsection{An Example on $\mathbb{T}^{n}$}

Theorem 7.6. Consider the standard flat torus $\mathbb{T}^{n}$. For every eigenvalue $\lambda$ there exists an eigenfunction $\varphi_{\lambda}$ on $\mathbb{T}^{n}$, a nodal domain $\mathcal{A}_{\lambda}$ and a ball $B$ of radius $\sim 1$ such that

$$
\frac{\operatorname{Vol}\left(\mathcal{A}_{\lambda} \cap B\right)}{\operatorname{Vol}(B)} \leq \frac{C_{3}(n)}{(\sqrt{\lambda})^{n-1}},
$$

where $\mathcal{A}_{\lambda} \nsubseteq \mathbb{B}$ and $\mathcal{A}_{\lambda} \cap \frac{1}{2} B \neq \emptyset$.

Proof. Let $\mathbb{T}^{n}=\mathbb{R}^{n} / \mathbb{Z}^{n}$ be the standard flat torus parametrized by the standard coordinates $\left(x_{1}, x_{2}, \ldots x_{n}\right)$, where $0 \leq x_{j}<1$. Let $k$ be an integer, and let $\varphi_{k}=\Pi_{j=1}^{n-1} \sin 2 \pi k x_{j} . \varphi_{k}$ is an eigenfunction corresponding to the eigenvalue $\lambda_{k}=4(n-1) k^{2} \pi^{2}$. Each nodal domain has cross sections in normal direction to the $x_{n}$-axis of area $<c / k^{n-1}$. Hence, if we take a ball $B \subseteq \mathbb{T}^{n}$ of radius 1 and we let $\mathcal{A}_{\lambda}$ be a nodal domain which contains the center of the ball, we have

$$
\frac{\operatorname{Vol}\left(\mathcal{A}_{\lambda} \cap B\right)}{\operatorname{Vol}(B)} \leq \frac{C(n)}{(\sqrt{\lambda})^{n-1}} .
$$




\section{References}

[Ahl73] L. V. Ahlfors, Conformal invariants: topics in geometric function theory, McGraw-Hill Book Co., New York, 1973, McGraw-Hill Series in Higher Mathematics.

[CM91] S. Chanillo and B. Muckenhoupt, Nodal geometry on Riemannian manifolds, J. Differential Geom. 34 (1991), no. 1, 85-91.

[DF88] H. Donnelly and C. Fefferman, Nodal sets of eigenfunctions on Riemannian manifolds, Invent. Math. 93 (1988), no. 1, 161-183.

[DF90] _ Growth and geometry of eigenfunctions of the Laplacian, Analysis and partial differential equations, Lecture Notes in Pure and Appl. Math., vol. 122, Dekker, New York, 1990, pp. 635-655.

[EK96] Y. Egorov and V. Kondratiev, On spectral theory of elliptic operators, Operator Theory: Advances and Applications, vol. 89, Birkhäuser Verlag, Basel, 1996.

[Ger66] Ju. K. Gerasimov, The three spheres theorem for a certain class of elliptic equations of high order and a refinement of this theorem for a linear elliptic equation of the second order, Mat. Sb. (N.S.) 71 (113) (1966), 563-585, Translation in: American Mathematical Society Translations. Series 2, Vol. 72: Fifteen papers on analysis, American Mathematical Society, Providence, R.I., 1968, pp. 135162.

[Gro96] H. Groemer, Geometric applications of Fourier series and spherical harmonics, Encyclopedia of Mathematics and its Applications, vol. 61, Cambridge University Press, Cambridge, 1996.

[GT83] D. Gilbarg and N. S. Trudinger, Elliptic partial differential equations of second order, second ed., vol. 224, Springer-Verlag, Berlin, 1983.

[Hör64] L. Hörmander, Linear partial differential operators, Second revised printing. Die Grundlehren der mathematischen Wissenschaften, Band 116, Springer-Verlag New York Inc., New York, 1964. 
[Hör94] N Notions of convexity, Progress in Mathematics, vol. 127, Birkhäuser Boston Inc., Boston, MA, 1994.

[Lan63] E. M. Landis, Some questions in the qualitative theory of secondorder elliptic equations (case of several independent variables), Uspehi Mat. Nauk 18 (1963), no. 1 (109), 3-62, Translation in Russian Math. Surveys 18 (1963), 1-62.

[Lu93] G. Lu, Covering lemmas and an application to nodal geometry on Riemannian manifolds, Proc. Amer. Math. Soc. 117 (1993), no. 4, 971-978.

[Man08] D. Mangoubi, On the inner radius of a nodal domain, Canad. Math. Bull. 51 (2008), no. 2, 249-260.

[MSG03] A. G. Monastra, U. Smilansky, and S. Gnutzmann, Avoided intersections of nodal lines, J. Phys. A 36 (2003), no. 7, 1845-1853.

[Nad76] N. S. Nadirashvili, A generalization of Hadamard's three circles theorem, Vestnik Moskov. Univ. Ser. I Mat. Meh. 31 (1976), no. 3, 39-42, Translation in: Moscow Univ. Math. Bull. 31 (1976), 30-32.

[Nad91] _ Metric properties of eigenfunctions of the Laplace operator on manifolds, Ann. Inst. Fourier (Grenoble) 41 (1991), no. 1, 259265.

[NPS05] F. Nazarov, L. Polterovich, and M. Sodin, Sign and area in nodal geometry of Laplace eigenfunctions, Amer. J. Math. 127 (2005), no. 4, 879-910.

Dan Mangoubi,

Einstein Institute of Mathematics, Hebrew University, Givat Ram, Jerusalem 91904, Israel

mangoubi@math.huji.ac.il 